\title{
COERCIVE SOLVABILITY OF THE NONLOCAL BOUNDARY VALUE PROBLEM FOR PARABOLIC DIFFERENTIAL EQUATIONS
}

\author{
A. ASHYRALYEV, A. HANALYEV, AND P. E. SOBOLEVSKII
}

Received 26 March 2001

The nonlocal boundary value problem, $v^{\prime}(t)+A v(t)=f(t)(0 \leq t \leq 1), v(0)=$ $v(\lambda)+\mu(0<\lambda \leq 1)$, in an arbitrary Banach space $E$ with the strongly positive operator $A$, is considered. The coercive stability estimates in Hölder norms for the solution of this problem are proved. The exact Schauder's estimates in Hölder norms of solutions of the boundary value problem on the range $\{0 \leq t \leq$ $\left.1, x \in \mathbb{R}^{n}\right\}$ for $2 m$-order multidimensional parabolic equations are obtained.

\section{Introduction}

We consider the following nonlocal boundary value problem for the differential equation

$$
\begin{gathered}
v^{\prime}(t)+A v(t)=f(t) \quad(0 \leq t \leq 1), \\
v(0)=v(\lambda)+\mu \quad(0<\lambda \leq 1),
\end{gathered}
$$

in an arbitrary Banach space with linear (unbounded) operator $A$. It is known (cf. [4]) that various nonlocal boundary value problems for the parabolic equations can be reduced to the boundary value problem (1.1). We obtain the coercive solvability of problem (1.1) in some function Banach space. The role played by coercive inequalities in the study of boundary value problems for elliptic and parabolic partial differential equations is well known (cf. [5, 6, 7]). Coercivity inequalities for the solutions of an abstract differential equation of parabolic type

$$
v^{\prime}(t)+A v(t)=f(t) \quad(0 \leq t \leq 1), \quad v(0)=v_{0},
$$

were established in the various norms of Banach spaces by Sobolevskii P. E. and $\mathrm{Da}$ Prato and their colleagues under the assumption that $A$ is a strongly positive operator, that is, $-A$ is the generator of the analytic semigroup $\exp \{-t A\}(t \geq 0)$

Copyright (C) 2001 Hindawi Publishing Corporation

Abstract and Applied Analysis 6:1 (2001) 53-61

2000 Mathematics Subject Classification: 65N, 47D, 34B

URL: http://aaa.hindawi.com/volume-6/S1085337501000495.html 
54 Coercive solvability of the nonlocal boundary value problem

of the linear bounded operators with exponentially decreasing norm (see [3]). In [1], the coercive solvability of a Cauchy problem (1.2) was established in $C_{0}^{\beta, \gamma}(E)(0 \leq \gamma \leq \beta, 0<\beta<1)$-the space obtained by completion of the space of all smooth $E$-valued functions $\varphi(t)$ on $[0,1]$ in the norm

$$
\|\varphi\|_{C_{0}^{\beta, \gamma}(E)}=\max _{0 \leq t \leq 1}\|\varphi(t)\|_{E}+\sup _{0 \leq t<t+\tau \leq 1} \frac{(t+\tau)^{\gamma}\|\varphi(t+\tau)-\varphi(t)\|_{E}}{\tau^{\beta}},
$$

that is established in the following theorem.

THEOREM 1.1. Let $A$ be a strongly positive operator in a Banach space $E$ and $f(t) \in C_{0}^{\beta, \gamma}(E)(0 \leq \gamma \leq \beta, 0<\beta<1)$. Then for the solution $v(t)$ in $C_{0}^{\beta, \gamma}(E)$ of the initial value problem (1.2), the coercive inequality

$$
\begin{aligned}
& \left\|v^{\prime}\right\|_{C_{0}^{\beta, \gamma}(E)}+\|A v\|_{C_{0}^{\beta, \gamma}(E)}+\left\|v^{\prime}\right\|_{C\left(E_{\beta-\gamma}\right)} \\
& \quad \leq M\left[\left\|v_{0}^{\prime}\right\|_{E_{\beta-\gamma}}+\beta^{-1}(1-\beta)^{-1}\|f\|_{C_{0}^{\beta, \gamma}(E)}\right]
\end{aligned}
$$

holds, where $M$ does not depend on $\beta, \gamma, v_{0}^{\prime}$, and $f(t)$.

THEOREM 1.2. Let $A$ be a strongly positive operator in a Banach space $E$ and $f(t) \in C_{0}^{\beta, \gamma}\left(E_{\alpha-\gamma}\right)(0 \leq \gamma \leq \beta \leq \alpha, 0<\alpha<1)$. Then for the solution $v(t)$ in $C_{0}^{\beta, \gamma}\left(E_{\alpha-\gamma}\right)$ of the initial value problem (1.2), the coercive inequality

$$
\begin{aligned}
& \left\|v^{\prime}\right\|_{C_{0}^{\beta, \gamma}\left(E_{\alpha-\beta}\right)}+\|A v\|_{C_{0}^{\beta, \gamma}\left(E_{\alpha-\beta}\right)}+\left\|v^{\prime}\right\|_{C\left(E_{\alpha-\gamma}\right)} \\
& \quad \leq M\left[\left\|v_{0}^{\prime}\right\|_{E_{\alpha-\gamma}}+\alpha^{-1}(1-\alpha)^{-1}\|f\|_{C_{0}^{\beta, \gamma}\left(E_{\alpha-\beta}\right)}\right]
\end{aligned}
$$

holds, where $M$ does not depend on $\alpha, \beta, \gamma, v_{0}^{\prime}$, and $f(t)$.

Here $C(E)$ stands for the Banach space of all continuous functions $\varphi(t)$ defined on $[0,1]$ with values in $E$ equipped with the norm

$$
\|\varphi\|_{C(E)}=\max _{0 \leq t \leq 1}\|\varphi(t)\|_{E}
$$

and the Banach space $E_{\alpha}(0<\alpha<1)$ consists of those $v \in E$ for which the norm (see [3])

$$
\|v\|_{E_{\alpha}}=\sup _{z>0} z^{1-\alpha}\|A \exp \{-z A\} v\|_{E}+\|v\|_{E}
$$

is finite.

A function $v(t)$ is called a solution of problem $(1.1)$ in $C_{0}^{\beta, \gamma}(E)$ if the following conditions are satisfied:

(i) If the functions $v^{\prime}(t), A v(t) \in C_{0}^{\beta, \gamma}(E)$. 
(ii) If the function $v(t)$ satisfies the equation and the boundary condition (1.1).

From the existence of such solutions evidently follows that $f(t) \in C_{0}^{\beta, \gamma}(E)$ and $\mu \in D(A)$.

In the present paper, the coercive inequalities in the norms of the same spaces for the solutions of the boundary value problem (1.1) are obtained. The exact Schauder's estimates in Hölder norms of the solution of the boundary value problem on the range $\left\{0 \leq t \leq 1, x \in \mathbb{R}^{n}\right\}$ for $2 m$-order multidimensional parabolic equations are obtained.

\section{Coercive solvability in $C_{0}^{\beta, \gamma}(E)$}

It is known that an operator $A$ is strongly positive in $E$ if and only if $-A$ is the generator of the analytic semigroup $\exp \{-t A\}(t \geq 0)$ of the linear bounded operators in $E$ with exponentially decreasing norm when $t \rightarrow+\infty$, that is, the following estimates hold.

$$
\begin{array}{r}
\|\exp \{-t A\}\|_{E \rightarrow E} \leq M e^{-\delta t}, \quad t\|A \exp \{-t A\}\|_{E \rightarrow E} \leq M, \\
t>0, M>0, \delta>0 .
\end{array}
$$

For a strongly positive operator $A$ we have that

$$
\left\|(I-\exp \{-\lambda A\})^{-1}\right\|_{E \rightarrow E} \leq \frac{M}{1-e^{-\delta \lambda}}, \quad 0<\lambda \leq 1 .
$$

THEOREm 2.1. Let $A$ be a strongly positive operator in a Banach space $E$ and $f(t) \in C_{0}^{\beta, \gamma}(E)(0 \leq \gamma \leq \beta, 0<\beta<1)$. Then for the solution $v(t)$ in $C_{0}^{\beta, \gamma}(E)$ of the boundary value problem (1.1), the coercive inequality

$$
\begin{aligned}
& \left\|v^{\prime}\right\|_{C_{0}^{\beta, \gamma}(E)}+\|A v\|_{C_{0}^{\beta, \gamma}(E)} \\
& \quad \leq M\left[\|A \mu+f(\lambda)-f(0)\|_{E_{\beta-\gamma}}+\beta^{-1}(1-\beta)^{-1}\|f\|_{C_{0}^{\beta, \gamma}(E)}\right]
\end{aligned}
$$

holds, where $M$ does not depend on $\beta, \gamma, \mu$, and $f(t)$.

Proof. From the strong positivity of $A$, it follows that

$$
\begin{aligned}
v(t)= & \exp \{-t A\}(I-\exp \{-\lambda A\})^{-1}\left\{\mu+\int_{0}^{\lambda} \exp \{-(\lambda-s) A\} f(s) d s\right\} \\
& +\int_{0}^{t} \exp \{-(t-s) A\} f(s) d s
\end{aligned}
$$

for the solution of problem (1.1) in the space $C_{0}^{\beta, \gamma}(E)$ (cf. [4]). Using this 
56 Coercive solvability of the nonlocal boundary value problem

formula, we have that

$$
\begin{aligned}
v_{0}^{\prime}= & f(0)-A u(0) \\
= & (I-\exp \{-\lambda A\})^{-1} \int_{0}^{\lambda} A \exp \{-(\lambda-s) A\}(f(\lambda)-f(s)) d s \\
& -\left((I-\exp \{-\lambda A\})^{-1} A \mu+f(\lambda)-f(0)\right) \\
= & I_{1}+I_{2},
\end{aligned}
$$

where

$$
\begin{aligned}
& I_{1}=(I-\exp \{-\lambda A\})^{-1} \int_{0}^{\lambda} A \exp \{-(\lambda-s) A\}(f(\lambda)-f(s)) d s, \\
& I_{2}=-\left((I-\exp \{-\lambda A\})^{-1} A \mu+f(\lambda)-f(0)\right) .
\end{aligned}
$$

Then the proof of Theorem 2.1 follows from the inequality (1.4) and the estimate

$$
\left\|v_{0}^{\prime}\right\|_{E_{\beta-\gamma}} \leq M\left[\|A \mu+f(\lambda)-f(0)\|_{E_{\beta-\gamma}}+\beta^{-1}(1-\beta)^{-1}\|f\|_{C_{0}^{\beta, \gamma}(E)}\right] .
$$

Using formula (2.7) and the estimates (2.1), (2.2) for $z>0$, we have that

$$
\begin{aligned}
& z^{1-\beta+\gamma}\left\|A e^{-z A} I_{2}\right\| \\
& \leq\left\|\left(I-e^{-\lambda A}\right)^{-1}\right\|_{E \rightarrow E}\left\{z^{1-\beta+\gamma}\left\|A e^{-z A}(A \mu+f(\lambda)-f(0))\right\|_{E}\right. \\
& \left.\quad+z^{1-\beta+\gamma}\left\|A e^{-(z+\lambda) A}\right\|_{E \rightarrow E}\|f(\lambda)-f(0)\|_{E}\right\} \\
& \leq M\left\{\|A \mu+f(\lambda)-f(0)\|_{E_{\beta-\gamma}}+\frac{z^{1-\beta+\gamma} \lambda^{\beta-\gamma}}{z+\lambda}\|f\|_{C_{0}^{\beta, \gamma}(E)}\right\} \\
& \leq M\left\{\|A \mu+f(\lambda)-f(0)\|_{E_{\beta-\gamma}}+\|f\|_{C_{0}^{\beta, \gamma}(E)}\right\} .
\end{aligned}
$$

Therefore

$$
\left\|I_{2}\right\|_{E_{\beta-\gamma}} \leq M\left[\|A \mu+f(\lambda)-f(0)\|_{E_{\beta-\gamma}}+\|f\|_{C_{0}^{\beta, \gamma}(E)}\right] .
$$

Now we estimate $I_{1}$ in the norm $E_{\beta-\gamma}$. Using formula (2.6) and the estimates (2.1), (2.2), for any $z>0$ we obtain

$$
\begin{aligned}
& z^{1-\beta+\gamma}\left\|A e^{-z A} I_{1}\right\|_{E} \\
& \quad \leq\left\|\left(I-e^{-\lambda A}\right)^{-1}\right\|_{E \rightarrow E^{z^{1-\beta+\gamma}}} \int_{0}^{\lambda}\left\|A^{2} e^{-(z+\lambda-s)}\right\|_{E \rightarrow E}\|f(\lambda)-f(s)\|_{E} d s \\
& \quad \leq M z^{1-\beta+\gamma} \int_{0}^{\lambda} \frac{(\lambda-s)^{\beta} d s}{(z+\lambda-s)^{2} \lambda \gamma}\|f\|_{C_{0}^{\beta, \gamma}(E)} .
\end{aligned}
$$


If $z \leq \lambda$, then

$$
z^{1-\beta+\gamma} \int_{0}^{\lambda} \frac{(\lambda-s)^{\beta} d s}{(z+\lambda-s)^{2} \lambda \gamma} \leq z^{1-\beta} \int_{0}^{\lambda} \frac{d s}{(z+\lambda-s)^{2-\beta}} \leq \frac{1}{1-\beta} .
$$

If $z>\lambda$, then

$$
\begin{aligned}
z^{1-\beta+\gamma} \int_{0}^{\lambda} \frac{(\lambda-s)^{\beta} d s}{(z+\lambda-s)^{2} \lambda \gamma} & \leq z^{-\beta+\gamma} \lambda^{-\gamma} \int_{0}^{\lambda} \frac{d s}{(\lambda-s)^{1-\beta}} \\
& =\frac{1}{\beta}\left(\frac{\lambda}{z}\right)^{\beta-\gamma}<\frac{1}{\beta}
\end{aligned}
$$

Therefore, for any $z>0$, we have that

$$
z^{1-\beta+\gamma} \int_{0}^{\lambda} \frac{(\lambda-s)^{\beta} d s}{(z+\lambda-s)^{2} \lambda \gamma} \leq \frac{1}{\beta(1-\beta)} .
$$

From the last estimate and (2.11), it follows that

$$
\left\|I_{1}\right\|_{E_{\beta-\gamma}} \leq \frac{M}{\beta(1-\beta)}\|f\|_{C_{0}^{\beta, \gamma}(E)} .
$$

Using the estimates (2.10), (2.15), and the triangle inequality, we obtain the estimate (2.8). Theorem 2.1 is then proved.

Remark 2.2. Note that the spaces of smooth functions $C_{0}^{\beta, \gamma}(E)$ in which coercive solvability has been established depend on the parameters $\beta$ and $\gamma$. However, the constants in the coercive inequality (2.3) depend only on $\beta$. Hence, $\gamma$ can be chosen freely in $[0, \beta]$, which increases the number of function spaces in which problem (1.1) is coercively solvable. For example, it is important that problem (1.1) is coercively solvable in the Hölder space without a weight $(\gamma=0)$.

\section{Coercive solvability in $C_{0}^{\beta, \gamma}\left(E_{\alpha-\beta}\right)$}

THEOREM 3.1. Let $A$ be a strongly positive operator in a Banach space $E$ and $f(t) \in C_{0}^{\beta, \gamma}\left(E_{\alpha-\gamma}\right)(0 \leq \gamma \leq \beta \leq \alpha, 0<\alpha<1)$. Then for the solution $v(t)$ in $C_{0}^{\beta, \gamma}\left(E_{\alpha-\gamma}\right)$ of the boundary value problem (1.1), the coercive inequality

$$
\begin{aligned}
& \left\|v^{\prime}\right\|_{C_{0}^{\beta, \gamma}\left(E_{\alpha-\beta}\right)}+\|A v\|_{C_{0}^{\beta, \gamma}\left(E_{\alpha-\beta}\right)} \\
& \quad \leq M\left[\|A \mu+f(\lambda)-f(0)\|_{E_{\alpha-\gamma}}+\alpha^{-1}(1-\alpha)^{-1}\|f\|_{C_{0}^{\beta, \gamma}\left(E_{\alpha-\beta}\right)}\right]
\end{aligned}
$$

holds, where $M$ does not depend on $\beta, \gamma, \alpha$, and $f(t)$. 
58 Coercive solvability of the nonlocal boundary value problem

Proof. The proof of Theorem 3.1 follows from the inequality (1.5) and the estimate

$$
\left\|v_{0}^{\prime}\right\|_{E_{\alpha-\gamma}} \leq M\left[\|A \mu+f(\lambda)-f(0)\|_{E_{\alpha-\gamma}}+\frac{1}{\alpha(1-\alpha)}\|f\|_{C_{0}^{\beta, \gamma}\left(E_{\alpha-\beta}\right)}\right] .
$$

Using formula (2.7) and the estimates (2.1), (2.2) for any $z>0$, we have that

$$
\begin{aligned}
& z^{1-\alpha+\gamma}\left\|A e^{-z A} I_{2}\right\|_{E} \\
& \leq\left\|\left(I-e^{-\lambda A}\right)\right\|_{E \rightarrow E}\left\{z^{1-\alpha+\gamma}\left\|A e^{-z A}(A \mu+f(\lambda)-f(0))\right\|_{E}\right. \\
& \left.\quad+z^{1-\alpha+\gamma}\left\|A e^{-(z+\lambda) A}(f(\lambda)-f(0))\right\|_{E}\right\} \\
& \leq M_{1}\left[\|A \mu+f(\lambda)-f(0)\|_{E_{\alpha-\gamma}}+\frac{z^{1-\alpha+\gamma}}{(z+\lambda)^{1-\alpha+\beta}}\|f(\lambda)-f(0)\|_{E_{\alpha-\beta}}\right] \\
& \leq M_{1}\left[\|A \mu+f(\lambda)-f(0)\|_{E_{\alpha-\gamma}}+\frac{z^{1-\alpha+\gamma} \lambda^{\beta-\gamma}}{(z+\lambda)^{1-\alpha+\beta}}\|f\|_{C_{0}^{\beta, \gamma}\left(E_{\alpha-\beta}\right)}\right] .
\end{aligned}
$$

Since

$$
\frac{z^{1-\alpha+\gamma} \lambda^{\beta-\gamma}}{(z+\lambda)^{1-\alpha+\beta}} \leq 1
$$

we have that

$$
z^{1-\alpha+\gamma}\left\|A e^{-z A} I_{2}\right\|_{E} \leq M_{1}\left[\|A \mu+f(\lambda)-f(0)\|_{E_{\alpha-\gamma}}+\|f\|_{C_{0}^{\beta, \gamma}\left(E_{\alpha-\beta}\right)}\right],
$$

for any $z>0$, and it follows that

$$
\left\|I_{2}\right\|_{E_{\alpha-\gamma}} \leq M_{1}\left[\|A \mu+f(\lambda)-f(0)\|_{E_{\alpha-\gamma}}+\|f\|_{C_{0}^{\beta, \gamma}\left(E_{\alpha-\beta}\right)}\right] .
$$

Now we estimate $I_{1}$ in the norm $E_{\alpha-\gamma}$. Using formula (2.6) and the estimates (2.1), (2.2) for any $z>0$, we obtain

$$
\begin{aligned}
& z^{1-\alpha+\gamma}\left\|A e^{-z A} I_{1}\right\|_{E} \\
& \quad \leq\left\|\left(I-e^{-\lambda A}\right)^{-1}\right\|_{E \rightarrow E} z^{1-\alpha+\gamma} \int_{0}^{\lambda}\left\|A^{2} e^{-(z+\lambda-s)}(f(\lambda)-f(s))\right\|_{E} d s \\
& \leq M_{1} z^{1-\alpha+\gamma} \int_{0}^{\lambda} \frac{1}{(z+\lambda-s)^{2-\alpha+\beta}}\|f(\lambda)-f(s)\|_{E_{\alpha-\beta}} d s \\
& \leq M_{1} z^{1-\alpha+\gamma} \int_{0}^{\lambda} \frac{(\lambda-s)^{\beta} d s}{(z+\lambda-s)^{2-\alpha+\beta} \lambda \gamma}\|f\|_{C_{0}^{\beta, \gamma}\left(E_{\alpha-\beta}\right)} .
\end{aligned}
$$


If $z \leq \lambda$, then

$$
\begin{aligned}
z^{1-\alpha+\gamma} \int_{0}^{\lambda} \frac{(\lambda-s)^{\beta} d s}{(z+\lambda-s)^{2-\alpha+\beta} \lambda \gamma} & \leq \frac{z^{1-\alpha+\gamma}}{\lambda \gamma} \int_{0}^{\lambda} \frac{d s}{(z+\lambda-s)^{2-\alpha}} \\
& \leq \frac{z^{\alpha}}{(1-\alpha) \lambda^{\alpha}} \leq \frac{1}{1-\alpha}
\end{aligned}
$$

If $z \geq \lambda$, then

$$
z^{1-\alpha+\gamma} \int_{0}^{\lambda} \frac{(\lambda-s)^{\beta} d s}{(z+\lambda-s)^{2-\alpha+\beta} \lambda \gamma} \leq \frac{z^{-\alpha+\gamma}}{\lambda \gamma} \int_{0}^{\lambda} \frac{d s}{(\lambda-s)^{1-\alpha}}=\frac{\lambda^{\alpha-\gamma}}{\alpha z^{\alpha-\gamma}} \leq \frac{1}{\alpha} .
$$

Therefore, for any $z>0$ we have that

$$
z^{1-\alpha+\gamma} \int_{0}^{\lambda} \frac{(\lambda-s)^{\beta} d s}{(z+\lambda-s)^{2-\alpha+\beta} \lambda \gamma} \leq \frac{1}{\alpha(1-\alpha)}
$$

From the last estimate and (3.7), it follows that

$$
\left\|I_{1}\right\|_{E_{\alpha-\gamma}} \leq \frac{M}{\alpha(1-\alpha)}\|f\|_{C_{0}^{\beta, \gamma}\left(E_{\alpha-\beta}\right)} .
$$

Using the estimates (3.6), (3.11), and the triangle inequality, we obtain the estimate (3.2). This completes the proof of Theorem 3.1.

Remark 3.2. Using this approach we can obtain the same results for solutions of the general boundary value problem

$$
v^{\prime}(t)+A v(t)=f(t) \quad(0 \leq t \leq 1), \quad v(0)=\sum_{i=1}^{p} c_{i} v\left(t_{i}\right)+\mu,
$$

where $0<t_{1}<t_{2}<\cdots<t_{p} \leq 1$, if the operator $I-\sum_{i=1}^{p} c_{i} e^{-t_{i} A}$ has a bounded inverse in $E$.

\section{Applications}

We consider the boundary value problem on the range $\left\{0 \leq t \leq 1, x \in \mathbb{R}^{n}\right\}$ for $2 m$-order multidimensional differential equations of parabolic type

$$
\begin{aligned}
& \frac{\partial v(t, x)}{\partial t}+\sum_{|\tau|=2 m} a_{\tau}(x) \frac{\partial^{|\tau|} v(t, x)}{\partial x_{1}^{\tau_{1}} \cdots \partial x_{n}^{\tau_{n}}}+\delta v(t, x)=f(t, x) \quad(0 \leq t \leq 1), \\
& v(0, x)=v(\lambda, x)+\mu(x), \quad 0<\lambda \leq 1, x \in \mathbb{R}^{n}, \quad|\tau|=\tau_{1}+\cdots+\tau_{n},
\end{aligned}
$$

where $a_{r}(x), f(y, x)$ are given sufficiently smooth functions and $\delta>0$ is a 
60 Coercive solvability of the nonlocal boundary value problem

sufficiently large number. We will assume that the symbol

$$
B(\xi)=\sum_{|\tau|=2 m} a_{r}(x)(i \xi)^{r_{1}} \cdots(i \xi)^{r_{n}}
$$

of the differential operator of the form

$$
B=\sum_{|r|=2 m} a_{r}(x) \frac{\partial^{|r|}}{\partial x_{1}^{r_{1}} \cdots \partial x_{n}^{r_{n}}},
$$

acting on functions defined on the space $\mathbb{R}^{n}$, satisfies the inequalities

$$
0<M_{1}|\xi|^{2 m} \leq|B(\xi)| \leq M_{2}|\xi|^{2 m}<\infty,
$$

for $\xi \neq 0$.

Problem (4.1) has a unique smooth solution. This allows us to reduce the boundary value problem (4.1) to the boundary value problem (1.1) in the Banach space $E$ with a strongly positive operator $A=B+\delta I$ defined by (4.3). We give in Theorem 4.1 a number of corollaries to Theorems 2.1 and 3.1.

THEOREM 4.1. The solutions of the boundary value problem (4.1) satisfy the following coercive inequalities:

$$
\begin{aligned}
& \left\|\frac{\partial v}{\partial t}\right\|_{C_{0}^{\beta, \gamma}\left(C^{\varepsilon}\left(\mathbb{R}^{n}\right)\right)}+\sum_{|\tau|=2 m}\left\|\frac{\partial^{|\tau|} v}{\partial x_{1}^{\tau_{1}} \cdots \partial x_{n}^{\tau_{n}}}\right\|_{C_{0}^{\beta, \gamma}\left(C^{\varepsilon}\left(\mathbb{R}^{n}\right)\right)} \\
& \leq M(\varepsilon)\left[\left\|\sum_{|\tau|=2 m} a_{\tau}(x) \frac{\partial^{|\tau|} \mu(x)}{\partial x_{1}^{\tau_{1}} \cdots \partial x_{n}^{\tau_{n}}}-f(\lambda, x)+f(0, x)\right\|_{C^{2 m(\beta-\gamma)+\varepsilon}\left(\mathbb{R}^{n}\right)}\right. \\
& \left.+\frac{1}{\beta(1-\beta)}\|f\|_{C_{0}^{\beta, \gamma}\left(C^{\varepsilon}\left(\mathbb{R}^{n}\right)\right)}\right], \\
& 0<2 m(\beta-\gamma)+\varepsilon<1,0 \leq \gamma \leq \beta, 0<\beta<1, \\
& \left\|\frac{\partial v}{\partial t}\right\|_{C_{0}^{\beta, \gamma}\left(C^{2 m(\alpha-\beta)}\left(\mathbb{R}^{n}\right)\right)}+\sum_{|\tau|=2 m}\left\|\frac{\partial^{|\tau|} v}{\partial x_{1}^{\tau_{1}} \cdots \partial x_{n}^{\tau_{n}}}\right\|_{C_{0}^{\beta, \gamma}\left(C^{2 m(\alpha-\beta)}\left(\mathbb{R}^{n}\right)\right)} \\
& \leq M(\alpha, \beta, \gamma)\left[\left\|\sum_{|\tau|=2 m} a_{\tau}(x) \frac{\partial^{|\tau|} \mu(x)}{\partial x_{1}^{\tau_{1}} \cdots \partial x_{n}^{\tau_{n}}}-f(\lambda, x)+f(0, x)\right\|_{C^{2 m(\beta-\gamma)}\left(\mathbb{R}^{n}\right)}\right. \\
& \left.+\frac{1}{\beta(1-\beta)}\|f\|_{C_{0}^{\beta, \gamma}\left(C^{2 m(\alpha-\beta)}\left(\mathbb{R}^{n}\right)\right)}\right], \\
& 0<2 m(\alpha-\gamma)+\varepsilon<1,0 \leq \gamma \leq \beta, 0<\alpha<1 \text {, }
\end{aligned}
$$


where $M(\varepsilon)$ do not depend on $\beta, \gamma, \alpha, \mu(x)$, and $f(t, x)$ and $M(\alpha, \beta, \gamma)$ do not depend on $\mu(x)$, and $f(t, x)$. Here $C^{\varepsilon}\left(\mathbb{R}^{n}\right)$ is the space of functions satisfying a Hölder condition with the indicator $\varepsilon \in(0,1)$.

The proof of Theorem 4.1 is based on Theorems 2.1 and 3.1, the strong positivity of the operator $A$ in $C^{\varepsilon}\left(\mathbb{R}^{n}\right)$, the coercive inequality for the solution of the resolvent equation of the elliptic operator $A$ in $C^{\varepsilon}\left(\mathbb{R}^{n}\right)$, and equivalent of the norms in the spaces $E_{\beta}=E_{\beta}\left(A, C\left(\mathbb{R}^{n}\right)\right)$ and $C^{2 m \beta}\left(\mathbb{R}^{n}\right)$ when $0<\beta<1 / 2 m$ (see $[2,3])$.

\section{References}

[1] A. O. Ashyralyev, Coercive solvability of parabolic equations in spaces of smooth functions, Izv. Akad. Nauk Turkmen. SSR Ser. Fiz.-Tekhn. Khim. Geol. Nauk (1989), no. 3, 3-13. MR 90i:35150. Zbl 677.34005.

[2] A. O. Ashyralyev and P. E. Sobolevskii, Coercive stability of a multidimensional difference elliptic equation of $2 m$-th order with variable coefficients, Investigations in the Theory of Differential Equations (Russian), Minvuz Turkmen. SSR, Ashkhabad, 1987, pp. 31-43. CMP 1009419.

[3] _ Well-Posedness of Parabolic Difference Equations, Birkhäuser Verlag, Basel, 1994. MR 95j:65094.

[4] S. G. Krein, Linear Differential Equations in a Banach Space, Nauka, Moscow, 1966 (Russian).

[5] O. A. Ladyzhenskaya, V. A. Solonnikov, and N. N. Ural'tseva, Linear and Quasilinear Equations of Parabolic Type, Translations of Mathematical Monographs, vol. 23, American Mathematical Society, Rhode Island, 1967. MR 39\#3159b. Zbl 174.15403.

[6] O. A. Ladyzhenskaya and N. N. Ural'tseva, Linear and Quasilinear Elliptic Equations, Academic Press, New York, 1968. MR 39\#5941. Zbl 164.13002.

[7] M. I. Vishik, A. D. Myshkis, and O. A. Oleinik, Partial differential equations, Mathematics in USSR in the Last 40 Years, 1917-1957 (Russian), Fizmatgiz, Moscow, 1959, pp. 563-599.

A. Ashyralyev: Department of Mathematics, Fatih University, Istanbul, TURKEY

Current address: International Turkmen-Turkish University, Ashgabat, TURKMENISTAN

E-mail address: charyar@go.com

A. Hanalyev: Department of Applied Mathematics, Turkmen State University, Ashgabat, TURKMENISTAN

E-mail address: aashyr@fatih.edu.tr

P. E. SobolevskiI: Institute of Mathematics, Hebrew University, Jerusalem, ISRAEL

E-mail address:pavels@math.hiji.ac.il 


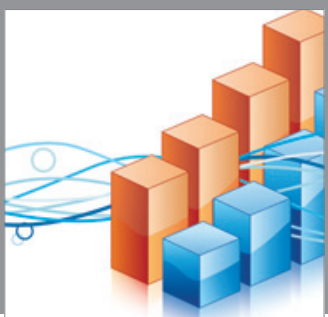

Advances in

Operations Research

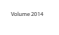

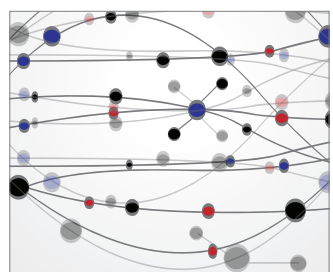

\section{The Scientific} World Journal
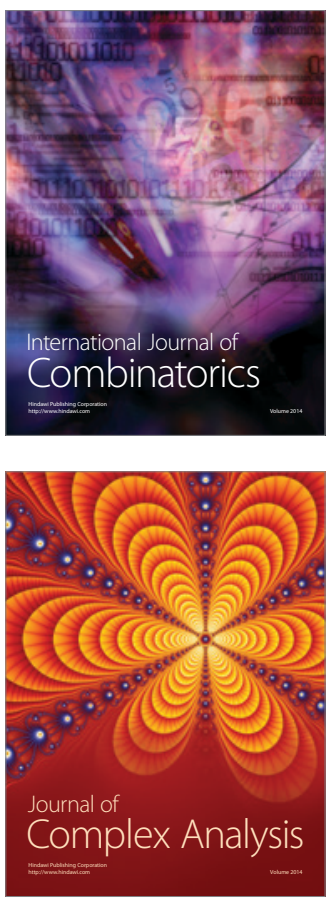

International Journal of

Mathematics and

Mathematical

Sciences
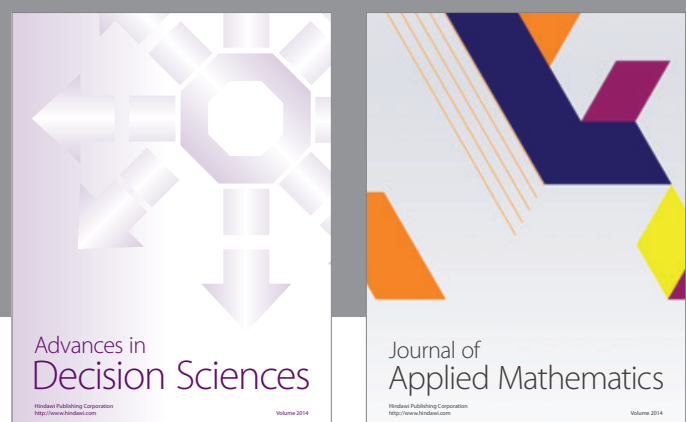

Journal of

Applied Mathematics
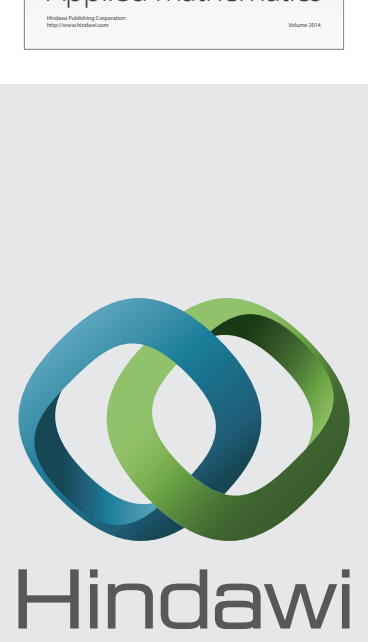

Submit your manuscripts at http://www.hindawi.com
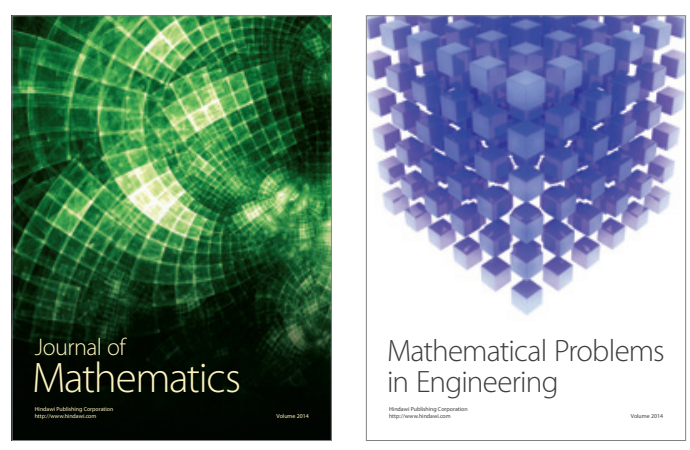

Mathematical Problems in Engineering
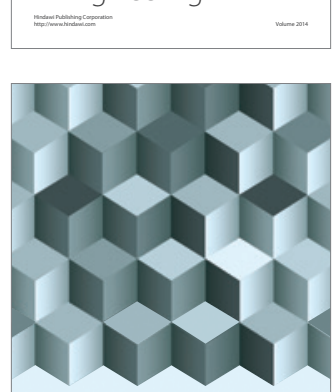

Journal of

Function Spaces
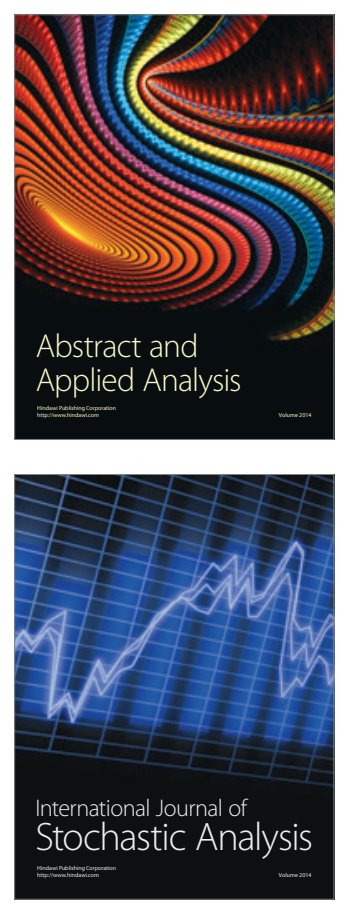

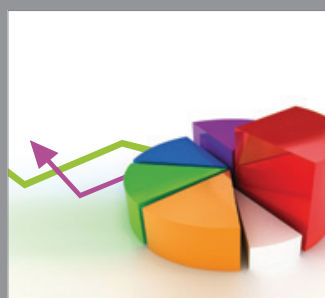

ournal of

Probability and Statistics

Promensencen
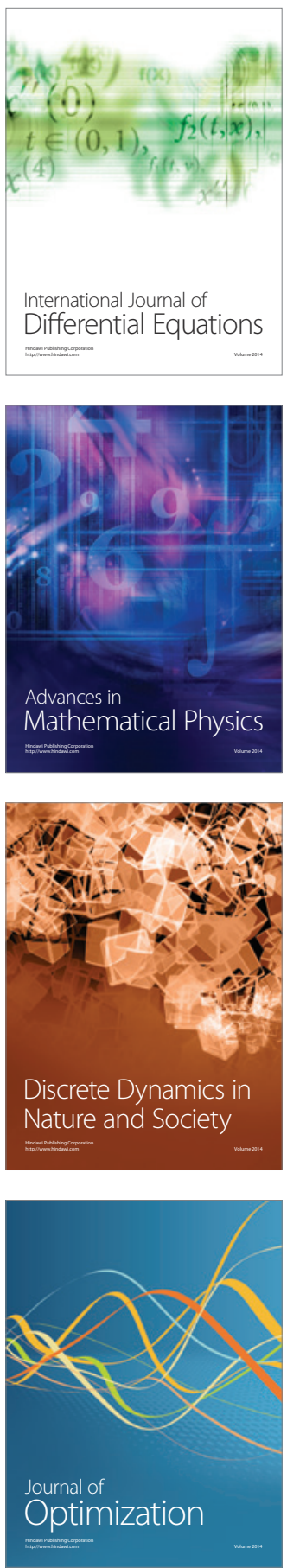